Journal of Engineering and Applied Sciences 14 (10): 3413-3421, 2019

ISSN: 1816-949X

(C) Medwell Journals, 2019

\title{
Muscles Activity Detection from EMG Signal of Human Leg Posture Afflicted by Foot Drop Disease
}

\author{
Yousif I. Al-Mashhadany \\ Department of Electrical Engineering, College of Engineering, University of Anbar, Ramadi, Iraq
}

\begin{abstract}
Surface Electromyography (SEMG) signal measurement technique in which an electrode connects to the surface of human muscle skin was produced from the mechanics of human muscle contraction. This study presents an off-line design for estimation of the actual joint angle of a human leg afflicted by foot drop disease. Flexion and extension of the leg are performed at low-speed and high speed movements. The design phases (two) first have real human-leg EMG signal measured by SEMG and processed by filtering, amplification and normalization with maximum amplitude, next an Artificial Neural Network (ANN) is trained to predict the joint angle from the parameters extracted from the SEMG signal. Three main parameters of the EMG signal are used in the prediction: the number of turns in a specific period, duration of signal repetition and signal amplitude. The ANN design includes two-speed (slow and fast) identification of the EMG signal and estimation of the knee joint angle by a recognition process that depends on the parameters of the real EMG signal measured from full leg-extension to full leg-flexion in slow motion $(3 \mathrm{sec})$ and fast motion $(1 \mathrm{sec})$. Root Mean Square (RMS) errors were calculated between the actual angle (trigonometric formula applied to human leg gives the real EMG signal measurement) and the angle predicted by the ANN. The design was simulated on MATLAB Ver. R2018a. Satisfactory results obtained show possible estimation of human leg joint angle with RMS errors of $(0.065)-(0.015)$ in fast leg flexion-extension and (0.018)-(0.0026) in slow leg flexion-extension.
\end{abstract}

Key words: Surface Electromyography (SEMG), Artificial Neural Network (ANN), foot drop, muscles activity, human muscle, estimation

\section{INTRODUCTION}

The Surface Electromyography (SEMG) is a non-invasive tool for investigation of skeletal muscle properties. The potentials recorded have relatively narrow bandwidth $(50-500 \mathrm{~Hz})$ and their amplitude is low $(50 \mu \mathrm{V}-5 \mathrm{mV})$. The signals have been used not only to monitor muscle behavior in rehabilitation programs but also, in mechanical control of prostheses. Correct prediction of the user-intended movement is important. SEMG suits such applications because it is noninvasive, simple to use and intrinsically relates to user intention. There are however, other useful variables especially related to proprioception, e.g., joint angle, limb position and force exerted (Delis et al., 2009; Geyer and Herr, 2010).

Development of active leg prosthesis with ankle and foot axes necessitates use of information other than SEMG signal. Myoelectric signals are thus used with variables related to proprioception, to improve reliability in closed-loop control systems. Figure 1 presents the typical main components of general myoelectric pattern recognition. SEMG signals are acquired by surface electrodes placed on the skin of the user's muscle $(\mathrm{sec})$. Signals from the electrodes are pre-amplified to sift out the small signals of interest and then amplified, filtered and digitized. The information is then transferred to a myoelectric controller (Pate et al., 2018).

Knee joint is one of the most complex synovial joints in the human body. Its main functions are to allow locomotive movement and a stable static. Knee joint mobility is indispensable to human locomotion. It helps correct foot orientation and positioning when overcoming ground irregularities. Knee articulation has three motions: flexion, rotation and the sliding of the patella. The knee joint has three functional compartments: medial, lateral and patello-femoral; they make the knee quite susceptible to injuries and chronic diseases-displacement, arthritis, ligament rupture and menisci separation. Most injuries to human ligaments are knee ligament injuries. The knee joint is surrounded by a joint capsule with ligaments strapping the inside and the outside of the joint (collateral ligaments) as well as crossing within the joint (cruciate ligaments) (Machado et al., 2010). 


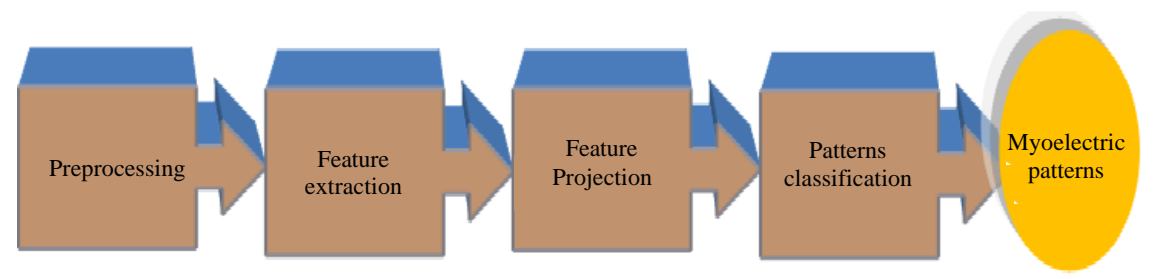

Fig. 1: Typical main components of a general myoelectric controller based on pattern recognition

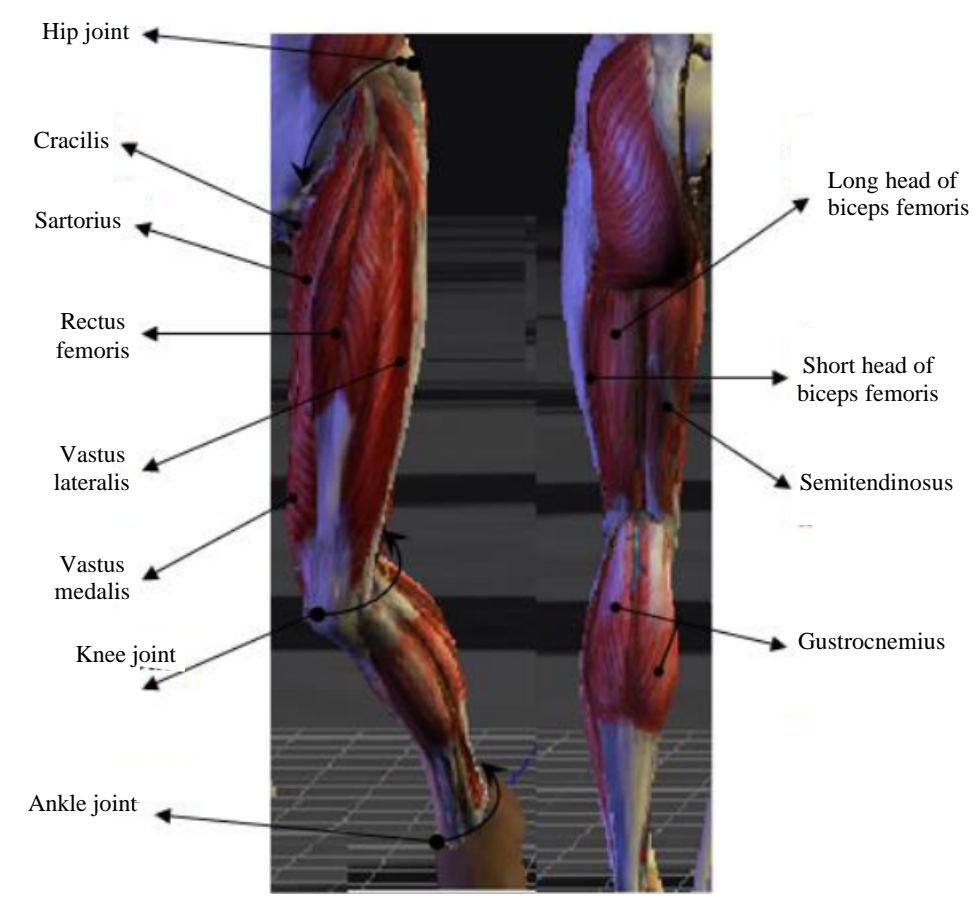

Fig. 2: Human leg muscles that cause flexion/extension of the knee joint (Mader, 2004)

Knee joint is one of the most complex synovial joints in the human body. Its main functions are to allow locomotive movement and a stable static. Knee joint mobility is indispensable to human locomotion. It helps correct foot orientation and positioning when overcoming ground irregularities. Knee articulation has three motions: flexion, rotation and the sliding of the patella. The knee joint has three functional compartments: medial, lateral and patello-femoral; they make the knee quite susceptible to injuries and chronic diseases-displacement, arthritis, ligament rupture and menisci separation. Most injuries to human ligaments are knee ligament injuries. The knee joint is surrounded by a joint capsule with ligaments strapping the inside and the outside of the joint (collateral ligaments) as well as crossing within the joint (cruciate ligaments) (Machado et al., 2010).

The muscles of the lower limbs are larger and more powerful than those of the upper limbs. They can be one of three groups (Fig. 2) (Ellis, 2006; Lopez et al., 2018) that move the thigh, the leg or the foot and toes.

\section{MATERIALS AND METHODS}

Movements of the knee: The principal movements of the knee are flexion and extension. The capsule attaches to the margins of the articular surfaces and above it to the suprapatellar bursa (between the lower femoral shaft and the quadriceps), posterior to the bursa under the medial head of the gastrocnemius and often, through it with the bursa under semimembranosus. It may also connect with the bursa under the lateral head of the gastrocnemius. The capsule is also perforated on its posterior by the popliteus which emerges the way the long head of the biceps bursts out of the shoulder joint.

The knee-joint capsule is reinforced on each side by the medial and lateral collateral ligaments, the latter passing to the head of the fibula, free of the capsule. On its anterior, the capsule is strengthened mostly by the ligamentum patellae which is strengthened on each side by the medial and lateral patellar retinacula which are 




Fig. 3: Location of SEMG on a human leg

expansions from the vastus medialis and lateralis. On its posterior, the tough oblique ligament arises as an expansion from the insertion of the semimembranosus and blends with the joint capsule (Bahadur and Rehman, 2018; Bu et al., 2003; Bida, 2005; Khan et al., 2016).

Real EMG signal measurement: The procedure real measurement of EMG signal in this research done by the flowchart is shown in Fig. 3, three main steps in this procedure as fellow.

Step 1; Select the positioning of the electrodes: Nine muscles up and down the knee joint affect knee movement. Clinical information and practical experience show the best position is the one resulting in maximum flexion/extension of the knee joint. Figure 3, S1 show the practical experiment in selecting the best position for two electrodes for the EMG signal to be obtained upon movement of the joint. This research used four muscles that least affects flexion/extension of the knee joint. The EMG signal recorded by the SEMG electrodes, therefore, must use four electrodes simultaneously (i.e., recording four channels). This was not possible practically, so, off-line technique was used instead, to estimate the joint angle through EMG signal (Reichl et al., 2010; Simsek, 2017).

Step 2; Real signal measurement by SEMG: The real SEMG signals were in this research recorded as such:

- (Spontaneous case) the knee joint relaxed and no movement in it

- From full extension to full flexion of the knee joint in slow motion $(3 \mathrm{sec})$ of the leg

- From full extension to full flexion of the knee joint in fast motion $(1 \mathrm{sec})$ of the leg

- The preceding two repeated with flexion angles 45 , $90,135^{\circ}$ or the maximum humanly achievable 
Figure 3, S2 is a photo of the SEMG signal for the main leg muscles that cause flexion/extension of the knee joint. They were as obtained using the instruments in Baghdad's Al-Yarmook Teaching Hospital. The experiment data was recorded by a single channel. This problem caused the design to be achieved offline where each movement needed four runs to obtain the full data.

Step 3; Filtering signal and amplification: Figure $3 \mathrm{~S} 3$ is a sample SEMG signal prepared through filtering and amplification; these were achieved through EMGLab Software which can process the real data recorded in the TRC data format by the Micromed instruments.

Design of estimation joint angle: The knee joint angle was in this research calculated by two methods. One uses the trigonometric relationship with human leg posture to get the knee-joint angle before measuring the real SEMG used in the second design estimating the joint angle. Figure 4 shows the human-leg geometry used in trigonometric form to calculate the joint angle. The calculations assume the radius of the cross-sectional area of the normal human leg aged 25 years to be $\mathrm{r}=13 \mathrm{~cm}$ (Simsek, 2017; Tarlochan et al., 2002):

$$
\mathrm{S}=\mathrm{R} \theta, \theta=\frac{\mathrm{S}}{\mathrm{R}} ; \theta \text { in rad; } \theta^{\circ}=\frac{\theta_{\mathrm{rad}^{*}} 180}{\pi}
$$
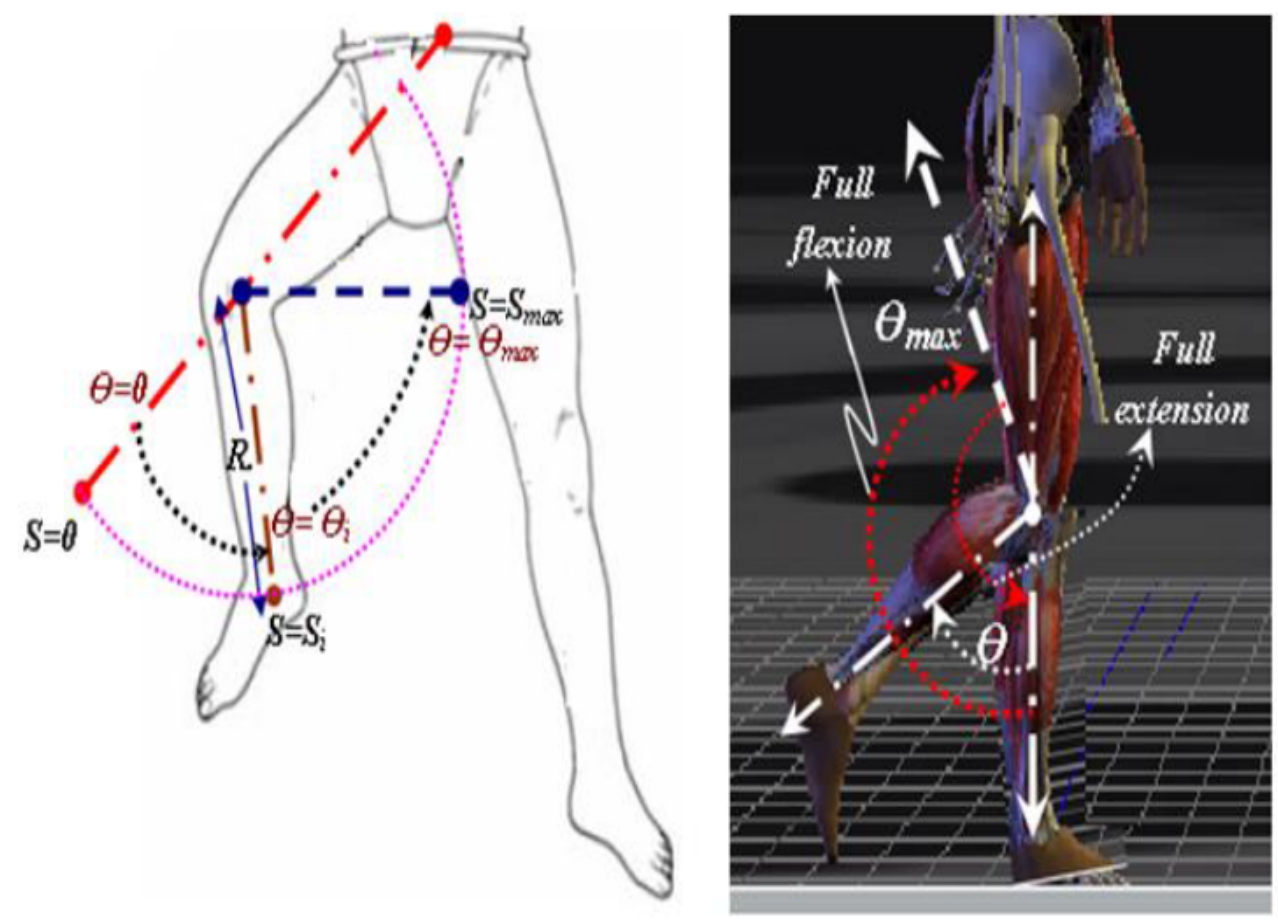

Fig. 4: Geometry calculation of the knee joint angle

Where:

$\mathrm{R}=$ The length of the lower leg (from the knee joint to the ankle joint)

$\mathrm{S}=$ The length of the arc from the initial position of the ankle joint to the second position of the ankle joint

$\theta=$ The angle of flexion/extension of the knee joint

The other method uses ANN (Fig. 5). The design has two main stages, next described.

The identification stage: Knee-joint movement studies show nine muscles effecting movement of the joint, four main muscles (vastus medialis, vastus lateralis for extension and Semitendinosus, Gustrocomis for flexion) causing joint angle in joint flexion/extension. The SEMG signals of the four muscles will be used in estimating the angle at leg movement.

This research used Recurrent Multilayer Perception (RMLP) Neural Network (NN) in identifying the SEMG signals of the four main muscles. Figure 6 block-diagrams the identification stage (Goodfellow and O'Connor, 1978). The NN structure is $(3 \times 2 \mathrm{R} \times 2(2))$ (Fig. 7), i.e., three units in layer 1 (the input layer), two recurrent units in layer 2 and 2 units in layer 3. The network has external feedback with 2 unit delays. Sigmoidal activation functions were used for all units in both the hidden and the output layers. The error function measuring the difference 


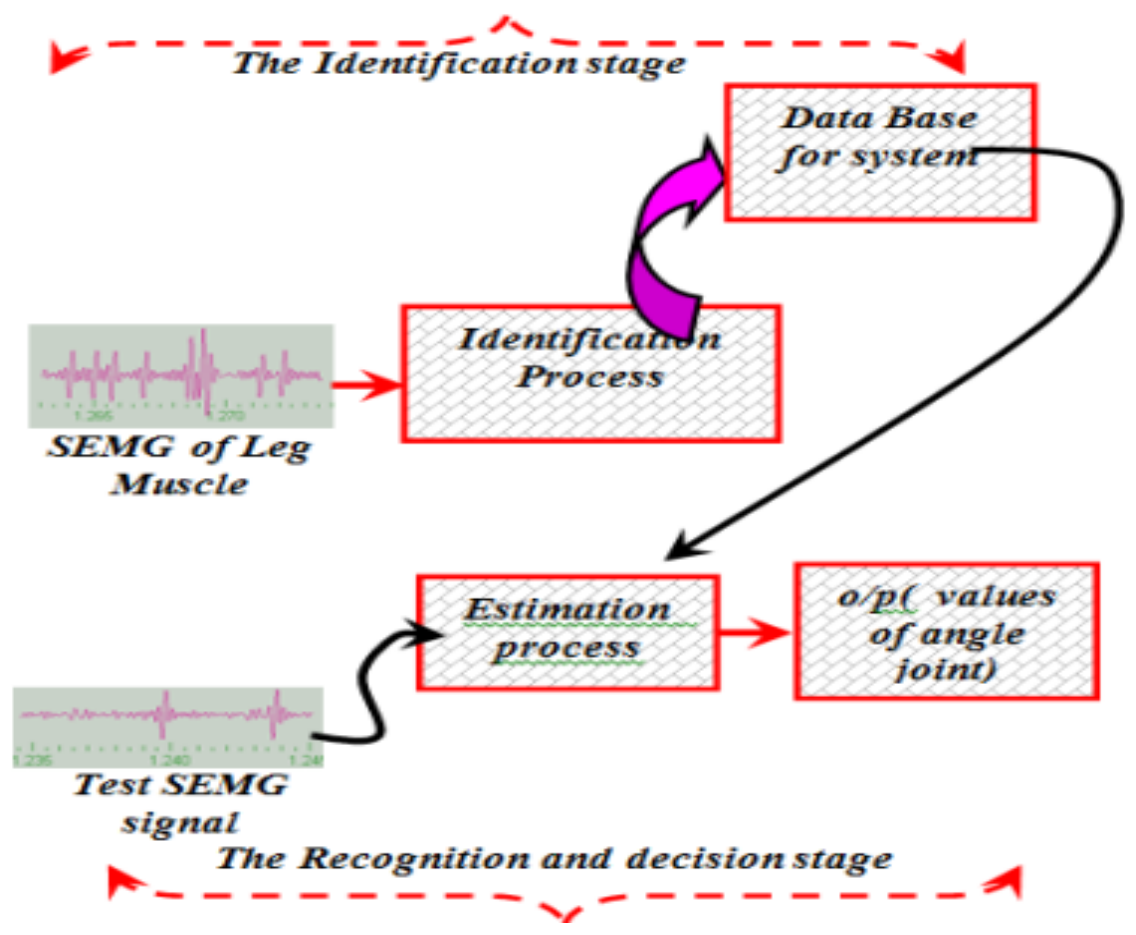

Fig. 5: The NN design for estimating knee joint angle

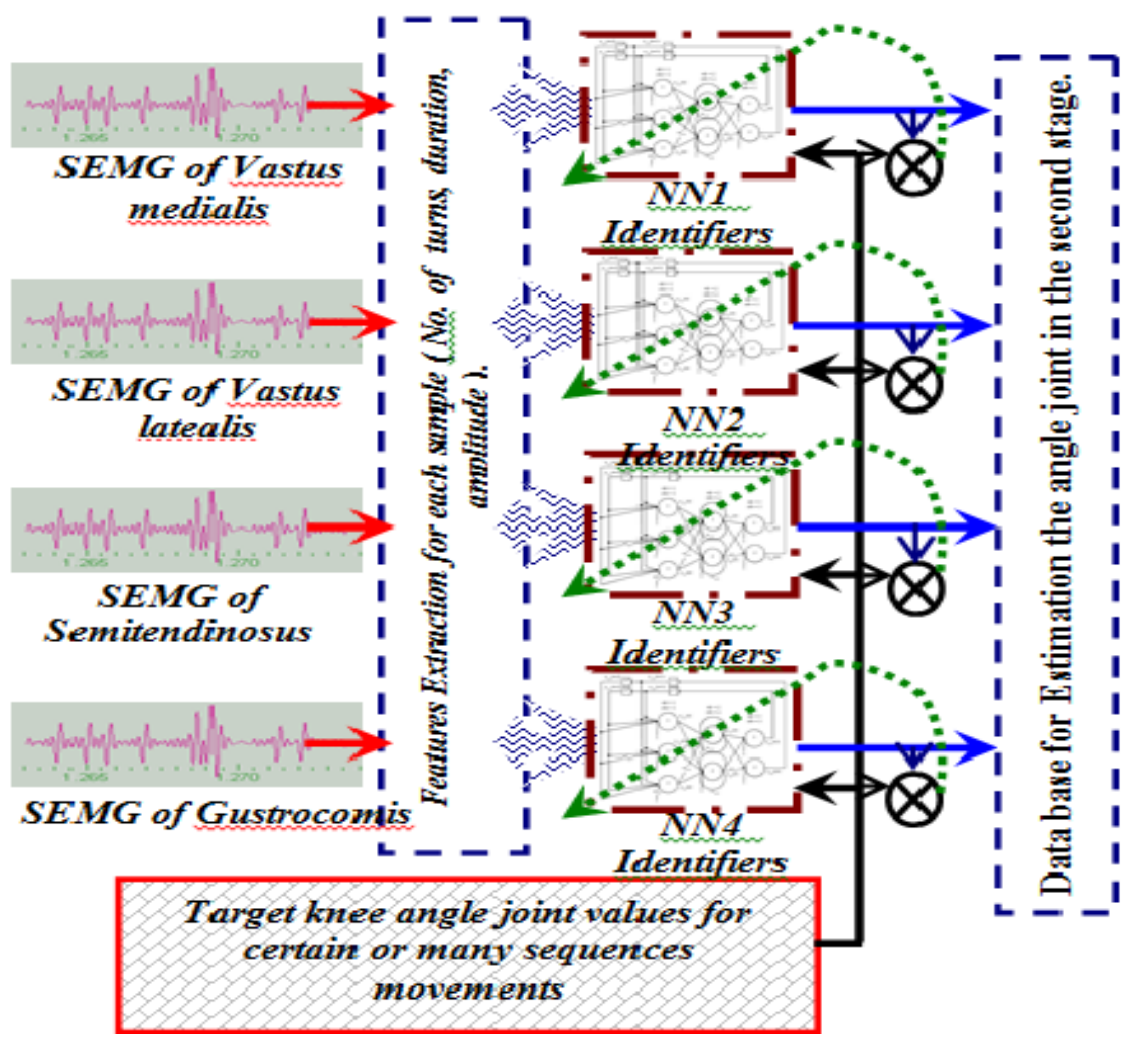

Fig. 6: The NN identification of the SEMG signal in human leg muscles 


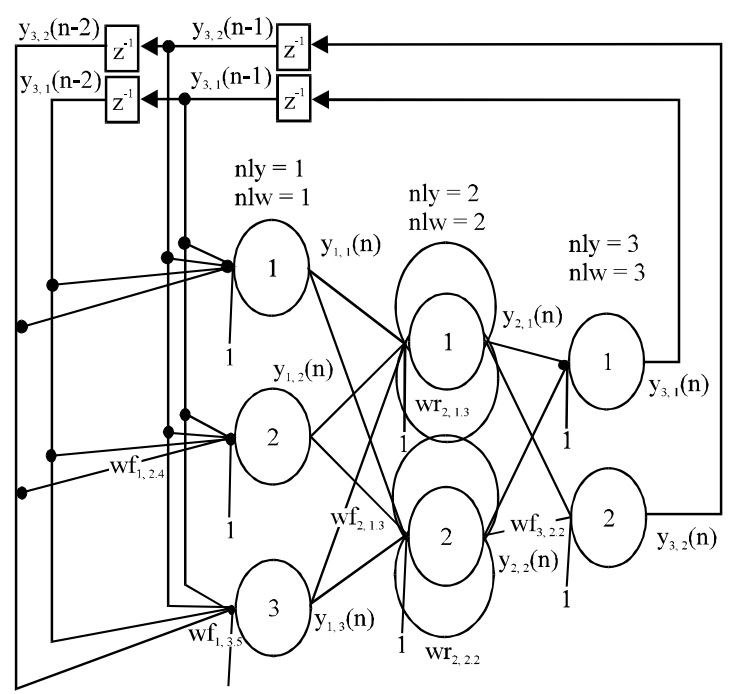

Fig. 7: Recurrent multilayer perception neural network with structure $(3 \times 2 \mathrm{R} \times 2(2))$ (Sato et al., 2018; Shrirao et al., 2009) between the neural net approximation and the desired trajectory (Al Mashhadany and Abd Rahim, 2013; Kulpa and Multon, 2005) is (Fig. 8):

$$
\left.\begin{array}{c}
\mathrm{E}(\mathrm{w})=\frac{1}{2} \sum_{\mathrm{n}=1}^{\mathrm{M}} \sum_{\mathrm{l}=1}^{\mathrm{L}}\left[\mathrm{d}_{1}(\mathrm{n})-\mathrm{y}_{1}(\mathrm{n})\right]^{2} \\
\theta_{\mathrm{f}_{1,2}}=\frac{\mathrm{A}_{1}{ }^{*} \theta_{\mathrm{f} \max }}{\mathrm{A}_{\max }} \\
\theta_{\text {flexion }}=\frac{\theta_{\mathrm{f}_{1}}+\theta_{\mathrm{f}_{2}}}{\mathrm{~A}_{\max }} \\
\theta_{\mathrm{E}_{1,2}}=\frac{\mathrm{A}_{\mathrm{i}}{ }^{*} \theta_{\mathrm{E} \max }}{\mathrm{A}_{\max }} \\
\theta_{\text {Extension }}=\frac{\theta_{\mathrm{E}_{1}}+\theta_{\mathrm{E}_{2}}}{2}
\end{array}\right\}
$$

The recognition and decision stage: The test signal recorded from the same type of muscles is used in

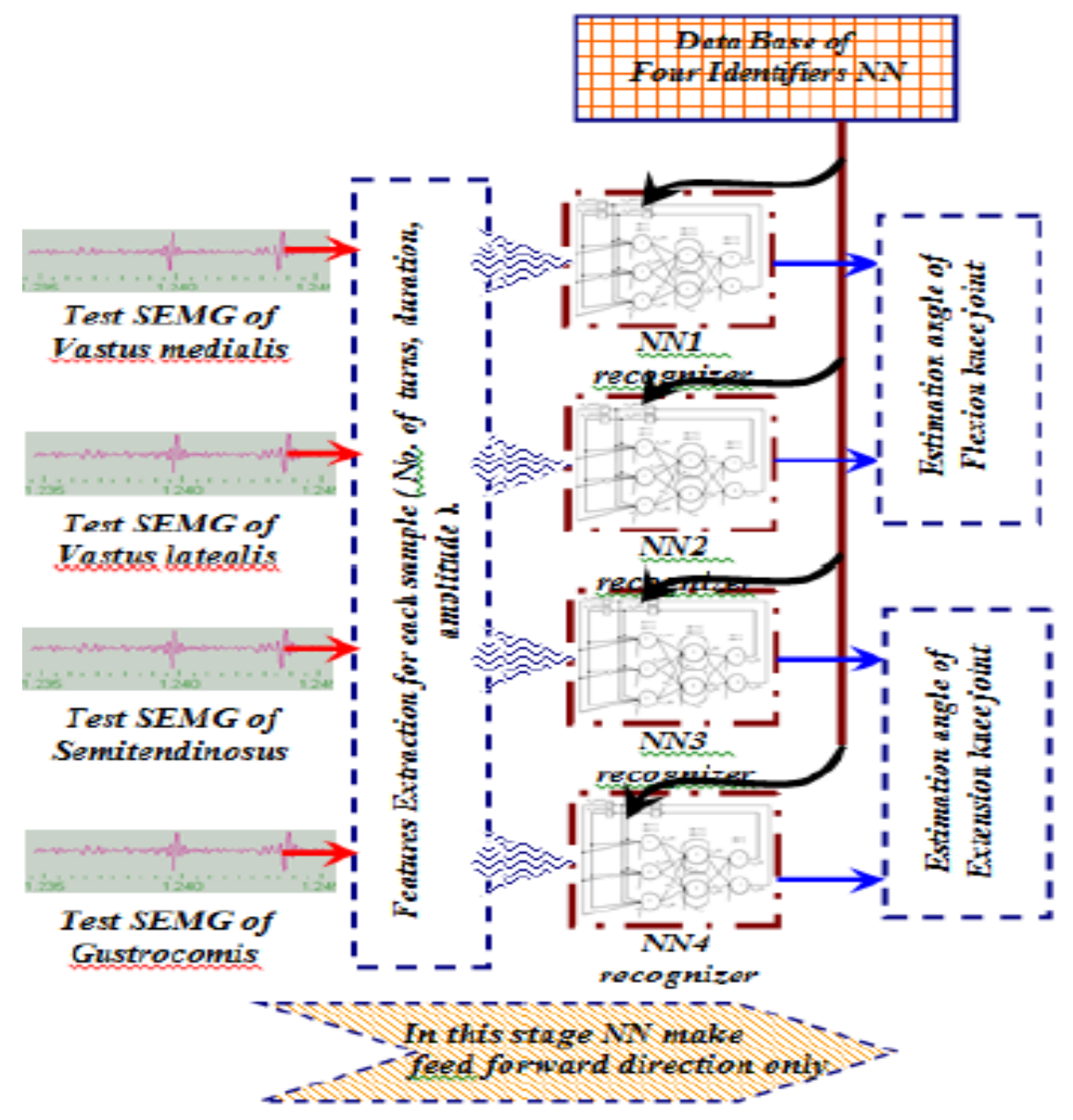

Fig. 8: Estimation of the knee-joint angle 
estimating the joint angle. The database isformatted by recording the SEMG signal of the muscles with joint angles 0 (no command from the brain), $\pi / 4, \pi / 2$ and $3 \pi / 4$ (maximum movement). After reconstructing the same features for the test signal will be entered to the same structure of RMLP-NN with identification data base by feed forward training only the output of these networks is recognizing the muscles and the amplitude of SEMG with each sample. Through the following relation with the SEMG signal over time and averaging the results from vastus medialis, vastus lateralis for extension and Semitendinosus, Gustrocomis for flexion, first the joint angle at flexion is obtained and then the complement angle (the extension angle). Figure 8 shows the second design stage in estimating knee-joint angle.

\section{RESULTS AND DISCUSSION}

Simulation results: The estimation design was simulated on MATLAB Var.R2018a. Figure 9 shows identification of human-leg SEMG signals relating to knee-joint flexion/extension. It shows almost zero error after about nine iterations, after which there was no local minimum or increase in error. This is therefore, the best results for identification post trial adjustment of the learning rate and momentum (Sato et al., 2018; Shrirao et al., 2009).

Low-speed leg movement: the leg was moved from full extension to full flexion with a speed of about $3 \mathrm{sec}$ per cycle. Figure 10 shows the estimated values for knee flexion/extension joint angle. Accurate joint angle was achieved by calculating the RMS error between the estimated values and the experiment value measured in the first stage of the design. The RMS error values were $0.065-0.015$.

High-speed leg movement: The same procedure as in low speed was repeated, Fig. 11 gives the results. The RMS errors were $0.018-0.0026$ at fast leg flexion/extension. Figure 12 shows the RMS values for two movement speeds (low and high) with the time for low speed being $180 \mathrm{sec}$ and for high speed $60 \mathrm{sec}$.

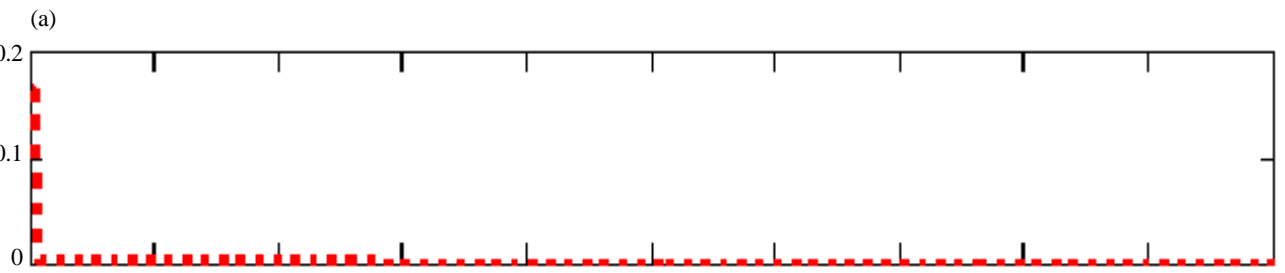

(b)

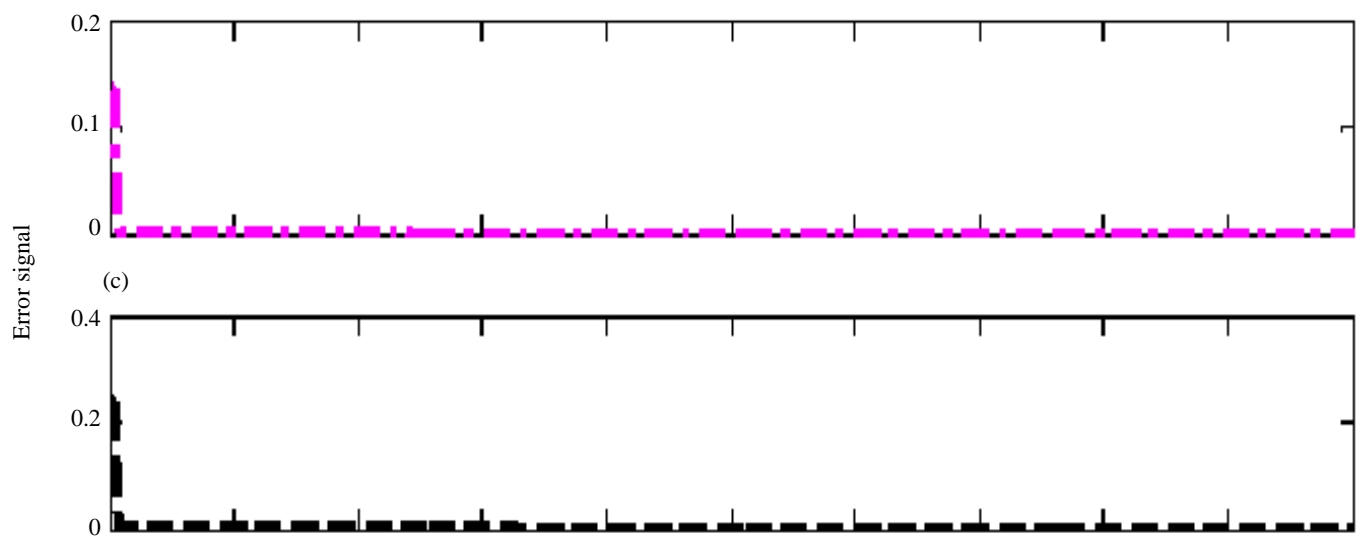

(d)

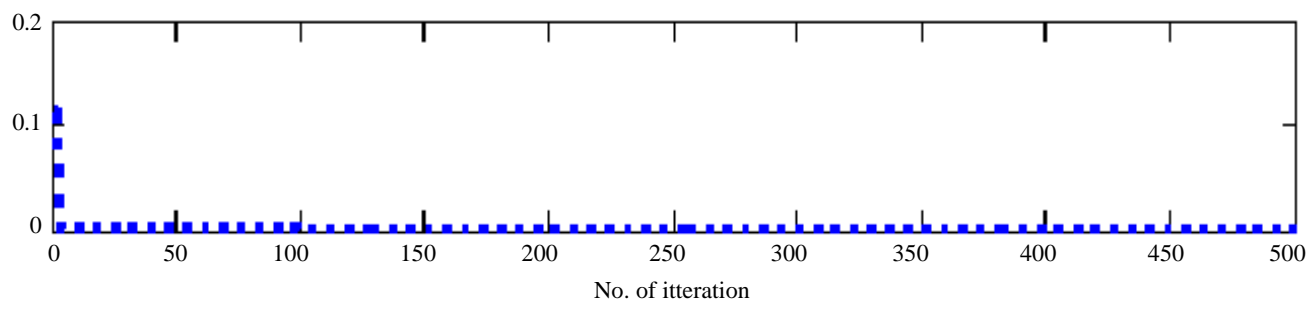

Fig. 9: a-d) Error signal for identification of the SEMG signal of four muscles 


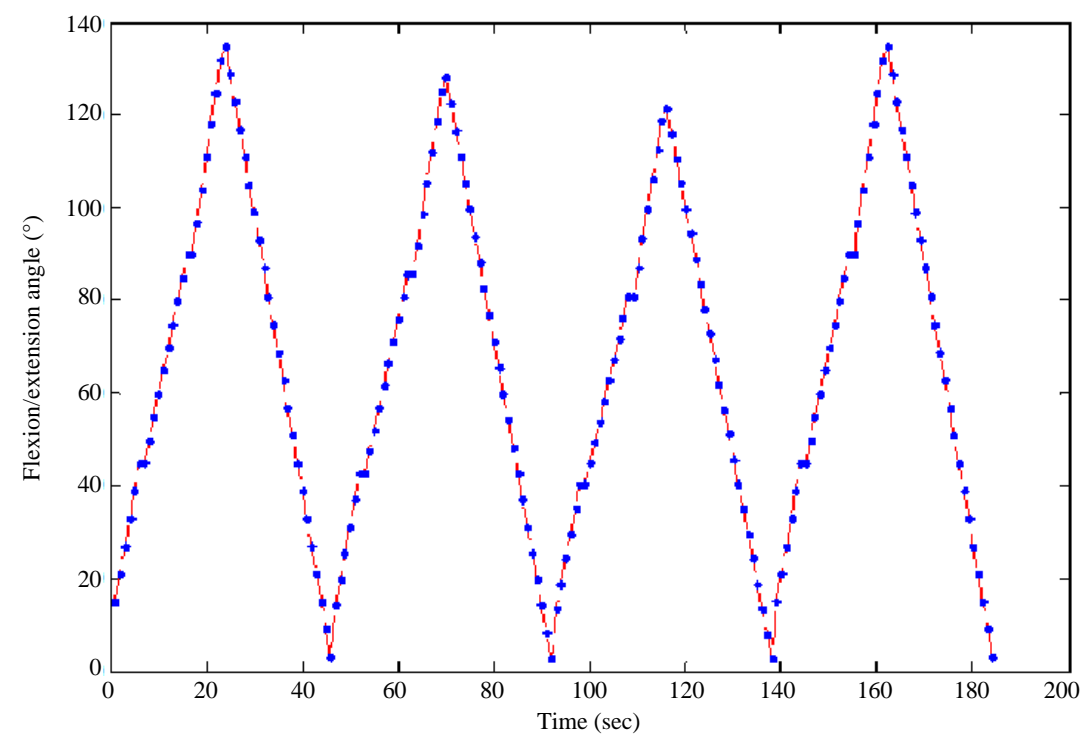

Fig. 10: Estimation of the knee-joint angle at low-speed leg movement

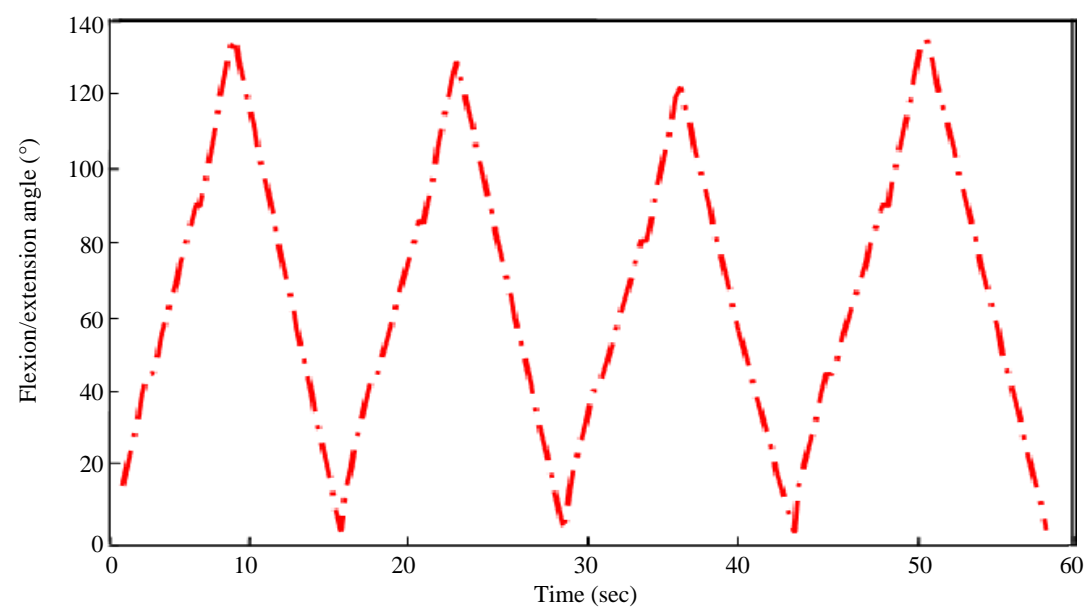

Fig. 11: Estimation of the knee-joint angle at fast leg movement

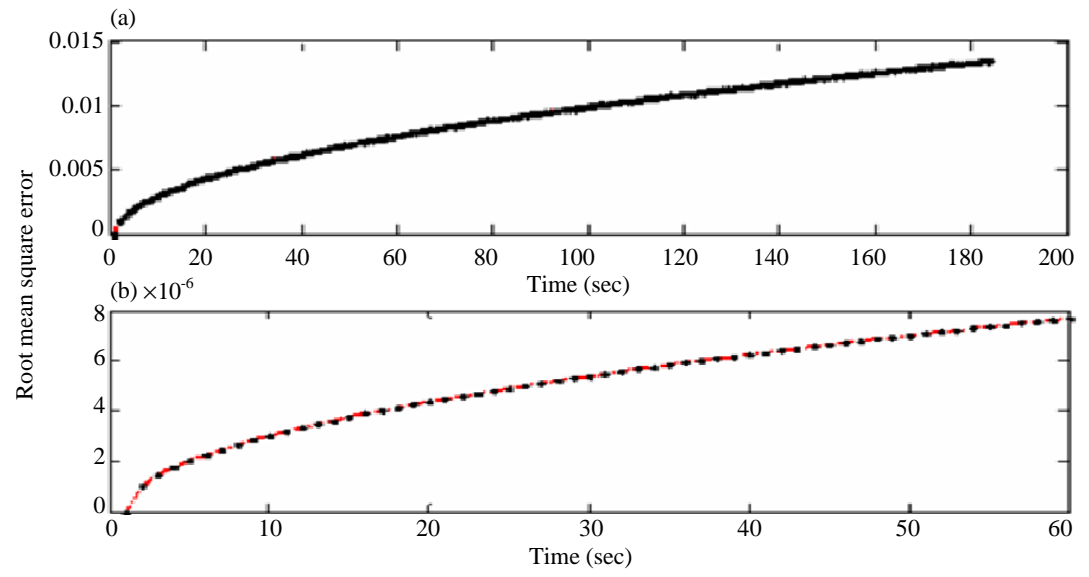

Fig. 12: a, b) RMS values for the two speeds of leg movement 


\section{CONCLUSION}

The results show the RMLP-NN capable of simple but accurate identification and solving of the IK of human leg. The highly accurate estimation of the knee-joint angle based on SEMG can benefit many applications. All these signals measurement under constrain of foot drop disease with this afflicting very difficult extracts the features from EMG signal to get the leg posture. Also, many critical coefficients in knee-joint movement must be considered in estimating the joint angle. The results were obtained off-line because multi-channel SEMG recording was not possible practically, so, the SEMG signals were individually recorded with the same command repeated each time. Available measurements, savable SEMG signals, online implementation and portability of the unit will expand its use to many applications.

\section{REFERENCES}

Al Mashhadany, Y.I. and N. Abd Rahim, 2013. Real-time controller for foot-drop correction by using surface electromyography sensor. Proc. Inst. Mech. Eng. Part H. J. Eng. Med., 227: 373-383.

Bahadur, R. and S. Rehman, 2018. A robust and adaptive algorithm for real-time muscle activity interval detection using EMG signals. Proceedings of the 11th International Joint Conference on Biomedical Engineering Systems and Technologies (BIOSTEC 2018) Vol 4, January 19-21, 2018, SciTePress, Funchal, MadeiraIsland, Portuga, ISBN:978-989-758-279-0, pp: 89-96.

Bida, O., 2005. Influence of electromyogram (EMG) amplitude processing in EMG-torque estimation. MSc Thesis, Worcester Polytechnic Institute, Worcester, Massachusetts.

Bu, N., O. Fukuda and T. Tsuji, 2003. EMG-based motion discrimination using a novel recurrent neural network. J. Intell. Inform. Syst., 21: 113-126.

Delis, A.L., J.L.A. de Carvalho, A.F. da Rocha, F.A.D.O. Nascimento and G.A. Borges, 2009. Development of a myoelectric controller based on knee angle estimation. Proceedings of the International Conference on Biomedical Electronics and Devices, January 14-17, 2009, SciTePress, Groningen, Netherlands, pp: 97-103.

Ellis, H., 2006. Clinical Anatomy: A Revision and Applied Anatomy for Clinical Students. 11th Edn., Wiley-Blackwell, Hoboken, New Jersey, USA., ISBN 13:9781405138048,
Geyer, H. and H. Herr, 2010. A muscle-reflex model that encodes principles of legged mechanics produces human walking dynamics and muscle activities. IEEE. Trans. Neural Syst. Rehabil. Eng., 18: 263-273.

Goodfellow, J. and J. O'Connor, 1978. The mechanics of the knee and prosthesis design. J. Bone Joint Surg., 60: $358-369$.

Khan, M., J. Singh and M. Tiwari, 2016. A multi-classifier approach of EMG signal classification for diagnosis of neuromuscular disorders. J. Bioengineer Biomed. Sci., 1: 1-5.

Kulpa, R. and F. Multon, 2005. Fast inverse kinematics and kinetics solver for human-like figures. Proceedings of the 5th IEEE-RAS International Conference on Humanoid Robots, December 5, 2005 , IEEE, Tsukuba, Japan, ISBN: 0-7803-9320-1, pp: 38-43.

Lopez, D.A.R., H.L. Correa, M.A. Lopez and J.E.D. Sanchez, 2018. Expert committee classifier for hand motions recognition from EMG signals. Ingeniare Chil. Mag. Eng., 26: 62-71.

Machado, M., P. Flores, J.P. Claro, J. Ambrosio and M. Silva et al., 2010. Development of a planar multibody model of the human knee joint. Nonlinear Dyn., 60: 459-478.

Mader, S.S., 2004. Understanding Human Anatomy and Physiology. 5th Edn., McGraw-Hill Higher Education, New York, USA., ISBN:9780072935172, Pages: 444.

Pate, M., N. Makaram, S. Balasubramanian and S. Ramakrishnan, 2018. Analysis of muscle fatigue using electromyography signals in gastrocnemius muscle during isometric plantar flexion. Intl. J. Biosci. Biochem. Bioinf., 8: 100-106.

Reichl, I., W. Auzinger, H.B. Schmiedmayer and E. Weinmuller, 2010. Reconstructing the knee joint mechanism from kinematic data. Math. Comput. Model. Dyn. Syst., 16: 403-415.

Sato, Y., T. Kawase, K. Takano, C. Spence and K. Kansaku, 2018. Body ownership and agency altered by an electromyographically controlled robotic arm. R. Soc. Open Sci., 5: 1-10.

Shrirao, N.A., N.P. Reddy and D.R. Kosuri, 2009. Neural network committees for finger joint angle estimation from surface EMG signals. Biomed. Eng. Online, 8: 1-11.

Simsek, D., 2017. Different fatigue-resistant leg muscles and EMG response during whole-body vibration. J. Electromyography Kinesiology, 37: 147-154.

Tarlochan, F., S. Ramesh and B.M. Hillberry, 2002. Dynamic analysis of the human knee. Biomed. Eng. Appl. Basis Commun., 14: 122-126. 